\title{
Labyrinthe
}

$7 \mid 2000$

Numéro 7

\section{Trajets de la différence sexuelle}

Éric Prenowitz et Laurent Dubreuil

\section{(2) OpenEdition}

Journals

Édition électronique

URL : http://journals.openedition.org/labyrinthe/774

DOI : $10.4000 /$ labyrinthe. 774

ISSN : 1950-6031

Éditeur

Hermann

Édition imprimée

Date de publication : 30 octobre 2000

Pagination : 89-101

Référence électronique

Éric Prenowitz et Laurent Dubreuil, « Trajets de la différence sexuelle », Labyrinthe [En ligne], 7 | 2000, mis en ligne le 02 mai 2005, consulté le 04 mai 2019. URL : http://journals.openedition.org/ labyrinthe/774 ; DOI : 10.4000/labyrinthe.774

Ce document a été généré automatiquement le 4 mai 2019.

Propriété intellectuelle 


\title{
Trajets de la différence sexuelle
}

\author{
Éric Prenowitz et Laurent Dubreuil
}

1 «Femmes, quelles différences?» - Est-ce une question? Qui la pose et à qui ? Sur qui ou sur quoi porte-t-elle? Si c'est une question sur les femmes, si elle demande à savoir quelles sont les différences des femmes, par rapport aux hommes, par exemple, si elle pose les femmes en tant que différentes, si elle suppose en se posant ainsi que ce sont les femmes qui portent la différence, alors elle s'inscrit dans l'une des plus vieilles traditions de la pensée occidentale. Sous des dehors d'une réciprocité symétrique - les hommes sont tout aussi différents des femmes que les femmes des hommes - «la différence» repose depuis toujours comme un fardeau sur les épaules des femmes : elles sont accusées et condamnées de différence. Le neutre et l'universel ont toujours été déclinés au masculin. À suivre la question dans cette direction, les femmes seraient d'abord des hommes défaillants. Il s'agirait alors d'un départ peu prometteur pour qui voudrait autant que possible faire un pas hors de ces traditions.

2 «Femmes, quelles différences ?» Peut-être que c'est aux femmes que se pose la question : femmes, quelles sont les différences, quelles sont vos différences? On pourrait s'étonner en ce cas que deux hommes se proposent d'y répondre. Disons d'abord, et non sans partager un certain étonnement, que d'une part notre « réponse » risque bien de rester une interrogation ouverte, et que d'autre part nous voudrions faire chanceler la tranquille confiance avec laquelle on se sert à tout moment de ces mots «homme » et "femme ». Nous ne doutons pas qu'il y en ait, des hommes et des femmes; nous doutons que l'on puisse dire en toute rigueur et même en principe ce que ces mots veulent dire. Question de langue, certes.

3 Tout ce que nous souhaitons proposer sur la question de la différence sexuelle se résume ainsi en une certaine sensibilité à la langue. Non pas qu'il faille trouver d'autres mots qui seraient plus proches de la vérité, même s'il est indispensable d'interroger et de faire travailler la langue, pour ne pas laisser « réifier » les mots. Mais il ne faut pas réifier les choses non plus, si l'on peut dire. Or, cela ne signifie pas, comme on a souvent cru comprendre, que tout est langage. Seulement qu'on n'est jamais sûr de savoir où s'arrête celui-ci. Ce ne sont pas quelques mots, en tout cas, qui vont définir, ici, la différence sexuelle. Mais si elle doit nous échapper, et pour notre plus grand bonheur, si elle ne se 
laisse pas saisir, rebelle qu'elle est à la forme du concept, elle est également notre expérience la plus quotidienne. Toutefois, son approche réclame la patience de la lecture voire une certaine disponibilité du lecteur à l'égarement d'une recherche. En d'autres termes, on n'écrit pas sur la différence sexuelle comme sur un autre sujet. Cette phrase, ce pourrait être notre titre ; elle convoque dans le champ de la différence les expériences croisées de l'écriture, de l'autre et du sujet, qui nous surprendront, que nous reprendrons toujours, et qui formeront l'étoffe des pages qui suivent.

Se doubler de vitesse Deux économies libidinales

4 Ici, déjà, sur ce seuil, essayons d'emblée de commenter notre démarche. Tentons un métadiscours qui anticipe sur son propre discours, pour le doubler de vitesse ou le faire doubler par un autre discours en surplomb : nous voilà déjà en plein dans le sujet. Pour commencer ce serait un geste qui résiste à l'interprétation ou à la lecture univoques. D'une part la tentation du métadiscours est celle de la maitrise, d'une compréhension qui vise à circonscrire non seulement le sujet traité, ici « la différence sexuelle », et d'abord en le nommant, mais également le traitement lui-même. D'autre part, le commentaire prêtant toujours le flanc au commentaire, la recherche d'une surenchère définitive est vouée à l'échec : l'interprétation n'a pas de point final. Par conséquent, loin d'assurer un pouvoir de maîtrise discursive, il revient à souligner qu'il y a toujours une part du feu à faire, qui échappe au déploiement du discours. Mais qu'y a-t-il de la différence sexuelle dans ces deux interprétations antinomiques d'une même démarche ? Disons par hypothèse et de manière préliminaire que la maîtrise serait du côté du « masculin » alors que le débordement serait du côté du "féminin». Ces deux adjectifs substantivés, neutres, notons-le, et donc masculins, désigneraient ici ce qu'on a appelé des « économies libidinales »: des comportements typiques du sujet, des choix conscients et/ou inconscients d'investissement affectif et psychique trahissant toujours des calculs du désir et du plaisir. Cette opposition économique ne reproduit pas une simple différenciation "naturelle», anatomique, entre homme et femme : au contraire, en se décalant de tout déterminisme essentialiste, elle permet de formuler de manière théorique la possibilité de quelque féminité chez un homme ou d'une part de masculinité chez une femme. De rendre compte du fait qu'il se trouve des hommes plus ou moins «maternels» et des femmes plus ou moins «phalliques». C'est faire suppléer à une opposition une opposition, ajouter ou laisser échapper le masculin et le féminin de l'homme et de la femme. Mais le décalage ainsi établi constitue déjà le premier pas d'une déconstruction de ce duel cruel homme/femme, vieux comme le monde phallocentrique. En fait cette opposition binaire a depuis toujours été hiérarchisée, privilégiant l'hommeUn alors même qu'elle semble consacrer le deux, et s'accompagnant des discours les plus archaïques sur une répartition des aptitudes innées (homme : présence, activité, culture, intelligible...; femme : absence, passivité, nature, sensible...). Mais posée ainsi de manière arrêtée et rigidement binaire, le système conceptuel des économies libidinales masculine et féminine garde encore quelque marque de la vieille opposition. On peut donc s'attendre à ce qu'il n'échappe pas à son tour à un travail de déconstruction. Il peut néanmoins, à un certain niveau et jusqu'à un certain point, dans certains contextes historiques ou politiques, à des fins pédagogiques notamment, encore servir. On s'y fiera donc pour un temps seulement. La typologie des économies libidinales ne peut être qu'une caractérisation par provision. En la suivant un instant, on peut dire que notre geste ici, précisément en ce qu'il résiste à l'interprétation - est-ce que notre tentative méta-discursive trahit une économie libidinale masculine ou féminine ? - est déjà, avant même d'aborder la question de la différence sexuelle, travaillé par elle. 
5 Nous n'y échappons pas. La différence sexuelle n'est pas au fond un sujet ou un objet sur lequel nous pouvons écrire : c'est elle aussi qui écrit chaque fois que nous nous mettons à écrire.

Les champs et la place de la différence

6 En guise de commentaire, donc, formulons deux engagements, ou deux souhaits, apparemment hétérogènes sinon contradictoires. D’abord, notre démarche ne doit pas, elle doit ne pas se réduire à une prise de position dans tel ou tel champ constitué politique, théorique, académique ou institutionnel - même si «la différence sexuelle » dit tout cela, déjà, en ce moment, en France. Non pas pour fuir quelque responsabilité politique ou morale gênante mais dans l'espoir d'une chance : que nous puissions ainsi aborder, mais sans bord justement et sans exemple, la différence sexuelle comme une position qui ne se laisse pas prendre, car elle se sauve chaque fois qu'on s'en approche. Mais en même temps, quoiqu'en deuxième lieu, nous voudrions aussi montrer, exemples à l'appui, qu'une certaine interprétation de la différence sexuelle fournit, en effet, des outils analytiques permettant de " lire " de manière nouvelle, puissante, à notre avis nécessaire, un grand nombre de structures et événements qui marquent ces champs de manière indélébile.

7 Nous ne pourrons pas en effet avoir recours à un discours qui s'épuise dans sa dimension théorique, se laissant arraisonner et donc approprier sans reste par quelque lecture que ce soit. Propos risqués, qui n'auront pas leur place à l'intérieur d'une discipline académique donnée, pas plus que dans un thème de revue, fût-elle labyrinthique. Le labyrinthe, voilà déjà qui nous donne le la : un enfermement ouvert, et d'autant plus redoutable, objet fractal avant la lettre qui subvertit l'opposition du dedans et du dehors au moyen d'une ligne de frontière qui s'étire par invagination sans jamais cesser de séparer et qui fait à la longue place nette au dedans. Du tribut payé au Minotaure en chair fraîche au fil d'Ariane en passant par Dédale le père célibataire, on pourrait montrer que le labyrinthe dès le départ entretient un rapport privilégié avec le sexe, les rapports des sexes ou sexuels, la différence sexuelle. Ce serait peut-être un nom du lieu sans lieu à partir duquel entamer la recherche sinon l'étude de la différence sexuelle. En fait, pour ce qui concerne l'approche que nous préconisons, alliant poésie et pensée de manière à envisager entre autres choses une transformation politique mais surtout une transformation du politique ou de la politique dont on ne fera pas éternellement l'économie - comme ce fut le cas récemment avec la parité, sorte de cache-sexe constitutionnel, nécessaire sans doute puisqu'on en est là, mais qui ne remplacera pas un vrai investissement politique toujours à venir -, les lieux de recherche et d'inscription ne sont pas donnés. Ils sont donc largement à inventer. Cela reflète, certes, le côté enlevé de la démarche, qui doit rester interdisciplinaire et non-codifiable, mais aussi la grande résistance, notamment institutionnelle, qu'elle peut susciter. Ce n'est pas un hasard si l'engagement à ce jour le plus riche avec l'irréductible différence sexuelle se tisse dans les textes d'Hélène Cixous et de Jacques Derrida, deux écrivains «hors catégorie » qui ont toujours entretenu des rapports compliqués avec les labyrinthes des institutions universitaires françaises ${ }^{1}$.

Sexué ou sexuel ? Le sujet sexué

8 La différence sexuelle : est-ce la différence du « sexuel » ? c'est-à-dire une différence de la «chose sexuelle », comme si c'était une chose, et par rapport à une autre chose qui ne serait pas sexuelle, n'aurait pas ou ne serait pas sujet à la différence sexuelle ? Est-ce qu'il y a quelque chose qui « précède » la différence sexuelle ? Est-ce que la différence sexuelle 
constitue une différence parmi tant d'autres ? ou est-ce qu'elle jouit d'un statut originaire, comme si elle était une sorte de différence matrice ou première qui donnerait le jour à toutes les autres, la condition de possibilité de toute différence constituée, à commencer par celle de la nature et de la culture?

Disons d'abord que la constitution du sujet est de part en part sexuelle ou sexuée. Il n'y a pas de sujet qui ne soit pas sujet à la différence sexuelle. Celle-ci ne vient pas après pour surimprimer de son sceau un sujet déjà constitué. Ce qui rend problématique notre quasidéfinition des économies libidinales qui voulait en faire des "comportements du sujet " et supposait en effet le principe d'un sujet sans différence sexuelle. Mais si l'analyse trouve ici une limite pour ainsi dire interne nous interdisant de désengager une réalité objective («sexe anatomique») et une constitution psychique ou sociale («gender»), cette limite ne nous oblige pas à adopter une attitude empirique ou relativiste, c'est-àdire à décréter face à la résistance de cet enchevêtrement à l'analyse qu'il n'y a pas de différence, qu'il n'y a que « sexe » ou " gender », biologie ou culture. Nos corps ne sont pas de purs fantasmes, même à l'âge des interventions chirurgicales permettant de « changer de sexe » de manière de plus en plus « vraisemblable » ou « réaliste ». En fait, tout ce que nous voudrons développer ici laisse à penser que la question de savoir ce que signifie changer de sexe doit rester ouverte, qu'à ce sujet il n'y a pas de vérité ou de réalité qui puisse permettre de trancher sans passer par une lecture de la différence sexuelle. Il n'est pas indifférent que nous soyons nés avec tel ou tel corps, même si «nous » ne faisons jamais l'expérience d'un rapport objectif, désincarné ou incorporel à notre corps. Celui-ci nous arrive avant nous. Nous ne sommes pas là, mais nous le recevons néanmoins en héritage. Et notre corps maintenant, dès que nous pouvons le penser ou formuler une phrase à son sujet, n'est pas tout à fait le même. Nous y sommes pour quelque chose. C'est précisément ce que nous faisons avec ce corps donné qui constitue le champ de la différence sexuelle. D'autant plus que «notre corps» n'a de réalité qu'inscrite, aucune nature qui ne soit pas toujours déjà reprise, labourée dans quelque champ culturel ou social, cosigné par l'autre à notre endroit mais avant nous : circoncision, par exemple, et toutes les marques, blessures, cicatrices, soins ou guérisons "physiques» et " psychiques ", car notre corps (nous) est raconté autant qu'incisé, avant même que nous sachions parler, marqué dans toutes ses peaux à mémoire.

De l'acte médico-légal

Or, contre ce jeu ouvert à tous les changements de sexe se déploie une histoire surdéterminée d'assignation dans la définition de l'identité sexuée. Prenons par exemple l'encadrement de la naissance dans les sociétés occidentales, le "C'est un garçon », ou le "C'est une fille " proférés par l'autorité clinique. L'enfant naît et reçoit sa première assignation. C'est un énoncé performatif : il décrit et il agit inséparablement. Autrement dit il s'agit d'un acte médico-légal, qui s'inscrit dans la scientificité médicale et dans l'enregistrement civil. C'est le premier geste de l'acculturation, une sorte d'autopsie primordiale qui tranche pour sexu(alis)er et situer dans l'opposition le nourrisson qui n'est pas encore un individu ou un sujet. Celui-ci aura toujours déjà été anatomisé en rapport avec un organisme de savoir, et non pas avec une incompressible réalité brute. Il n'est pas inintéressant de se pencher sur des discours de transsexuels qui recourent avec une violence au moins comparable à l'acte médico-légal pour "se délivrer $»^{2}$. La « liberté » obtenue est pourtant un assentiment sans équivoque à l'assignation anatomobiologique qui se double souvent d'affirmations du type de "J'étais une femme prisonnière d'un corps d'homme", ou l'inverse. Comme s'il fallait repasser par une 
naissance chirurgicale, comme s'il fallait la concordance des éventuels extérieur et intérieur, comme s'il fallait éviter d'être, par exemple, une femme dans un homme. Une pensée de la différence sexuelle se propose, quant à elle, l'expérience sans assignation exclusive d'une déconstruction de l'opposition que constitue la sexuation, et qui revient toujours à instaurer ou à restaurer le un. La question de l'homme-et-de-la-femme ne prend alors de signification qu'à la limite des devenirs, là où nous porterons plusieurs voix et plusieurs peaux, les masculines avec les féminines, ou l'inverse, innombrablement.

$\mathrm{Ni}$ essentialisme ni relativisme Les pièges de l'opposition

11 Nous ne nous contentons pas en effet de la triste alternative qui opposerait le "sexe" comme détermination « biologique », " anatomique » au " gender » comme détermination "culturelle», avatar de la sempiternelle opposition-piège de l'essentialisme et du relativisme. Il n'y a pas de choix à faire entre l'un et l'autre.

L'essentialisme pour qui un homme est un homme et une femme une femme, avec toutes les caractéristiques qui en découlent, imprègne encore notre société. Il s'est confondu, dans sa tradition scientifico-rationaliste, avec l'histoire même de l'Occident, une des histoires qu'il se raconte, mais combien puissante, comme une histoire de progrès ${ }^{3}$. En supposant des essences identifiables, il doit poser une réalité objective, c'est-à-dire universelle ou universalisable. Il fait appel à un méta-discours (universel, objectif) à partir duquel on peut justifier toutes les violences de domination. C'est nous ${ }^{4}$, dit-il en somme - et ce «nous » fait déjà foi, là aussi par quelque effet performatif : le narrateur qui dit «nous » est par définition celui qui porte le métadiscours. C'est nous, dit-il donc, qui avons fait le plus de progrès sur le chemin de la vérité ; et il n'y a qu'une vérité, c'est l'essentiel, elle est universelle, seulement nous y avons un accès privilégié - à la vérité scientifique, bien sûr, mais du coup à celle morale, culturelle, de la « civilisation ». Quant à la misogynie, ce n'était qu'une conséquence «naturelle » du « fait » que les hommes se trouvent par essence du côté de l'activité, de l'intelligence, de la force, etc., tandis que les femmes sont du côté de la passivité, du sentiment, de la faiblesse æ de la différence.

Le relativisme, qui peut servir parfois, comme au début de ce texte, par sa contestation du métadiscours d'autorité, amène des remises en cause violentes de tout ce qui avait l'apparence des données ou de la nature dans l'essentialisme. Par l'intensité de son doute, le relativiste réfute la croyance dans les essences. Le mouvement d'interrogation, le renoncement à la rassurante fixité des essences, la reconnaissance de l'indécidable qu'aucune raison ne tranchera, - voilà autant d'éléments figurant dans le relativisme et qu'il est utile de reprendre. Mais vers quel sens ? Rendre l'absolu des identités naturelles et l'universel choses relatives devrait pousser la pensée vers le lien de relation, et, par là, l'accès à l'autre.

Or, au-delà du moment d'ébranlement de l'essentialisme, ce qui paraît s'être imposé communément sous la qualification du relativisme se refuse l'autre. Pour le « relativiste relativiste", tout se vaudrait, tout reviendrait au même. Il ne peut y avoir de l'autre radicalement autre. C'est le triomphe paradoxal de l'identité. À ce stade, on peut aller vers une espèce de renoncement caricatural. Une forme banale en est l'individualisme courant qui admet comme pivot l'individu, c'est-à-dire l'un indivisible, l'un perturbé par aucun autre, qui échapperait de la sorte au phénomène de la sexuation. Comme le sujet transcendantal que la différence sexuelle ne concernait pas mais qui servait d'appui à une anthropologie misogyne. Par un chemin de traverse, le relativisme rejoint subrepticement l'essentialisme, les deux sont solidaires d'une unique opposition, ils s'entretiennent mutuellement. 
Le sexe culturellement défini un certain culturalisme, qui s'articule autour de la notion de gender. Par ce courant d'idées, développé aux États-Unis, en croissance en Europe, on affirme solennellement que l'identité sexuelle est déterminée culturellement, qu'elle est construite. Il semble qu'on soit ainsi à l'opposé de toute naturalisation. Et pourtant, ce « relativisme à visage humain » en vient toujours et très vite à consentir à une détermination sous-jacente, extérieure aux champs culturels et sociaux dont elle a l'air de faire grand cas. Les expressions le disent, on est ainsi du côté de la «différence des sexes " qui présume l'existence des sexes entre lesquelles passeraient des différences. Mais d'où vient la certitude d'identification de ces «sexes" ? Par «l'empiricité irréductible», dirait, par exemple, Geneviève Fraisse ${ }^{5}$. Ce curieux mot d'empiricité désigne une distinction évidente, physique, organique, biologique, scientifique, essentialiste entre l'homme et la femme à laquelle se surajouteraient les constructions sociales. En l'occurrence, le culturalisme masque la répartition la plus traditionnelle, la plus rudimentaire avec laquelle elle fait alliance. En prime, l'auteur nous offre une empiricité, qui considère l'expérience comme un élément transparent ou révélateur (les hommes et les femmes, ça se voit, pardi !) et, avec le suffixe en -icité, rabat sur elle une connotation philosophicoscientifique : le néologisme louche vers les vérités de l'évidence et de la connaissance.

La différence sexuelle : dans le vif du sujet

$\mathrm{Ni}$ essentialisme, ni relativisme, une pensée de la différence sexuelle passe par les chemins de l'universel et du relatif mais se passe de leurs conclusions. Il s'agit d'un neutre (ni sexe ni gender, ni- ni-) qui doit être sexué, sans réduction à une opposition binaire. Nous ne craignons pas le relativisme, car nous savons qu'il n'est pas une fin en soi, nous n'avons pas besoin de nous acoquiner aux régressions définitoires pour trouver un peu de paix. L'effondrement interne de la positivité empirique n'aboutit pas à la chimère d'un masculin, d'un féminin entrelacés et réunis. Il n'entraîne pas un retrait du champ social, ce que semblent souvent redouter les partisans du gender, mais il doit aider à une autre position, une action de la pensée.

Ainsi, l'évocation des procédés de la sexuation ne détourne pas du sort des femmes et de la différence aujourd'hui et maintenant.

On pourrait le voir sur un exemple précis, dans la rapidité de notre scène quotidienne. On s'attardera un peu sur le combat pour le rétablissement de la gynécologie de ville, qui a pris en France une ampleur notable ${ }^{6}$. Depuis les années 1980, on ne forme plus de praticiens libéraux spécialistes. Il y a à cela des raisons immédiates qui sont dénoncées par celles, par ceux qui militent pour la gynécologie médicale. Une tension interne au corps médical est sensible et il en va là de l'intérêt des hospitaliers contre les médecins libéraux. Évidemment, des raisons économico-politiques sont en jeu, et les gouvernements successifs, malgré leur souci proclamé de la santé publique, démantèlent progressivement le système de soins au nom des restrictions budgétaires (la formation coûte toujours trop cher). Au-delà des principes généraux qui pourraient en découler et motivent sans doute l'action de beaucoup (lutte des petits contre les gros, contre l'économisme, pour la défense des conquêtes sociales, etc.), nous voudrions nous intéresser à la signification de ce combat par rapport aux questions de la différence sexuelle. (Et ce d'autant plus que la scène médicale est un des espaces dont l'enjeu tourne autour de la différence, comme nous le disions plus tôt.) 

gynécologie au profit de l'obstétrique ${ }^{7}$, et donc de l'assignation du corps féminin à la reproduction et de la redéfinition de l'identité sexuée par la clinique de l'accouchement. De manière complémentaire, l'augmentation massive de mastectomies et d'hystérectomies qu'entraînera la fin de la gynécologie s'accorde avec la définition médico-légale du sexe. Alors qu'on se fonde sur la science de l'organe pour catégoriser, la fixation d'une identité sexuée sur la foi de l'anatomie est donnée à la naissance et reprise chirurgicalement. Les femmes qui subissent « la totale » sont totalement niées en tant que femmes - selon la définition rudimentaire et fixiste que leur accorde la société. L'ablation ne les libère pas des déterminations, et, au contraire, accroît leur enfermement dans le champ clos des sexuations "objectives ${ }^{8}$. En ce sens l'enfermement est le symptôme sociétal de ladite définition basique à laquelle personne n'échappe.

Mais la stratégie universaliste, dissimulée éventuellement sous le masque étonnant du culturalisme, peut aussi bien réclamer le rétablissement de la gynécologie. Face à la réalité insuppressible du corps féminin, on reprend les bonnes vieilles explications de la tradition hippocrato-galénique : la femme est naturellement malade, en tout cas plus faible. «Différente ». Cette physiologie fragile réclamerait d'urgence des soins adaptés.

21 Malgré le clivage pratique, les adversaires sont d'accord sur l'essentiel : sur la positivité, durable et normative, de la définition sexuelle. De différence, point. Il faut pourtant que la différence sexuelle intervienne dans cette lutte.

Dans ce sens, œuvrer pour la gynécologie signifie combattre le dispositif essentialiste. Mais il convient de voir que l'appoint donné à un certain féminisme qui repousse la sexuation dans la construction mais la sauvegarde en son foyer naturel et médical est vigoureusement hostile à toute différence sexuelle. Qui doit s'entendre dans la création de l'acte et de l'interprétation, d'un seul bloc multiplié et fissuré. Si dans la direction d'une réussite politique ou pragmatique, il y a convergence des objectifs, cette union va forcément avec un écart insurmontable dont la mesure doit être prise à chaque instant ; et soulignée.

Cette première approche est surtout un éloignement progressif de l'opposition, de la disposition des sexes. Les murs tombent mais on ne sort jamais de la différence comme d'un dédale. Une écriture qui s'y confronte, autant qu'il se peut, ne peut avoir barre sur elle-même. Elle fait plutôt l'expérience de l'autre dans la différence de la différence... à l'infini, comme une lecture qui s'ouvre et ne se ferme pas. Un discours autre que celui des disciplines est à mener sur la différence sexuelle ; sur elle, un autre, d'autres discours que celui-ci sont à exprimer. Tout reste à faire et à prononcer, nous en sommes sûrs. Le plus important, après tout, était de dessiner dans ces lignes l'allure de la différence, son lent voyage fait de traversées accélérées. Voici la différence sexuelle qui ne connaît des sujets que leur trajet, la trajectoire contredisant et altérant sans cesse la sous-jacence immobile du subjectum. Voici la différence, elle file, elle passe, elle traverse. La voici. 


\section{BIBLIOGRAPHIE}

ChILAND Colette, Changer de sexe, Paris, Odile Jacob, 1997.

CiXous Hélène, «Sorties », dans La Jeune née (avec Catherine Clément), Paris, Union Générale d'Éditions, 1975.

- «Contes de la différence sexuelle », dans Lectures de la différence sexuelle (Mara Negron, éd.), Paris, Des femmes, 1994.

DERRIDA Jacques, « Voice II », dans Points de suspension, Paris, Galilée, 1992.

— « Fourmis », dans Lectures de la différence sexuelle (M. Negron, éd.), Paris, Des femmes, 1994.

FRAISSE Geneviève, La Différence des sexes, Paris, Presses Universitaires de France, 1996.

MERCADER Patricia, L'Illusion transsexuelle, Paris, L'Harmattan, 1994.

\section{NOTES}

* Cet article prend son point de départ dans le travail mené au séminaire d'Hélène Cixous (" poétique de la différence sexuelle », Université Paris-VIII, Collège international de philosophie).

1. On pourrait songer plus spécialement à «Sorties » et « Contes de la Différence Sexuelle » de Cixous et à « Voice II » et « Fourmis » de Derrida.

2. Sur ces discours naturalisants, on pourra se reporter aux exemples cités par Colette Chiland (Changer de sexe, p. 130 sq., 179 sq. et passim) et aux (auto)biographies analysées par Patricia Mercader (dans son chapitre VII, voir surtout p. 249 sqq.).

3. Il manque ici la dimension chronologique de ces phénomènes, les flux et reflux des pensées. Dans les limites de cet article, on comprendra peut-être qu'on ait opté pour une perspective cavalière.

4. Cf. plus haut sur le métadiscours. Nous disons «nous », on l'aura remarqué, nous aussi. Il y a toujours le risque, dès que " nous " parlons, de parler pour l'autre, même pour l'autre en nous-même. Mais il y a plus qu'une façon d'encourir ce risque structural. Il s'agit d'abord, ici, de tenter de penser ce risque, surenchère qui gagne à perdre.

5. Voir sa Différence des sexes. Le quatrième chapitre (et en particulier les pages 49 sq.) est consacré à l'irréductibilité de cette " empiricité », au « butoir » qu'est le « fait sociologique et social ». Cf. également la conclusion (p. 125).

6. Une pétition du Comité de défense de la gynécologie médicale circule, qui a recueilli près d'un million de signatures.

7. Les dernières propositions gouvernementales orientent d'ailleurs les pétitionnaires vers un projet de fusion obstétrique-gynécologie.

8. Le parallèle est évident avec les transsexuels qui, dans le sens femme-homme, subissent la même opération, qui, dans leur cas et en complément d'autres actes chirurgicaux, est perçue comme re-naturalisante. 


\section{RÉSUMÉS}

Le présent texte se propose d'approcher, autant que faire se peut, les enjeux de la différence sexuelle - et cherche en particulier à dénouer les liens qu'entretient cette dernière avec l'écriture, l'autre et le sujet. Trois notions qui parcourent l'ensemble de l'article. Partant de ce qui s'écrit et se lit ici, le texte s'offre d'emblée à son commentaire et décrit les économies libidinales toujours déjà à l'œuvre dans les gestes philosophique ou théorique. Ces économies peuvent servir de première piste pour la différence et elles aident à la contestation des oppositions "naturelles». Il faudra les relever par un cran supplémentaire dans la déconstruction ; pour l'instant, elles permettent de dessiner le premier labyrinthe de la différence. Où l'on finit par trouver la nécessité d'un sujet renouvelé, d'un sujet sexué. C'est lui qui permet la déconstruction de l'essentialisme et le déplacement du relativisme, sans recourir à la combinaison de ces deux positions d'opposition repérée dans le leurre du "culturalisme ", cette affirmation de la construction sexuelle sur fond de positivité anatomique irréfutable. Preuve à l'appui avec une lecture de la différence sexuelle menée à partir d'une scène concrète et politique*.

Trips of sexual difference

This article is an attempt to introduce a certain approach to sexual difference. Beginning with its own reading the text adresses the question of its commentary. There is no metadiscurse possible and likewise no metasexual position is available from which to observe sexual difference. Sexual difference is already at work in any approach to sexual difference. This approach is therefore double and divided in itself. On the one hand is involved a deconstruction of the binary oppositions that have traditionnaly ruled over Western thought, and in particular the opposition of man to woman. A first step is to consider male and female «libidinal economies » wich are anchored in some anatomical reality. They therefore serve to contest the grip of so-called "natural oppositions ». Thus the arid alternative between essentialism and relativism need not dictate the limits of sexual difference. No more than a "culturalist» illusion in which the affirmation of sexuality as socially constructed force back upon fondation of irrefutable biological positivity. On the other hand an attempt is made to conduct our reading of sexual difference with respect to a concrete and political situation.

\section{AUTEURS}

\section{ÉRIC PRENOWITZ}

Éric Prenowitz va prochainement soutenir une thèse de doctorat en études féminines (à l'Université Paris-VIII) sous la direction d'Hélène Cixous. Il a traduit en anglais Mal d'archive de Jacques Derrida.

\section{LAURENT DUBREUIL}

Laurent Dubreuil dirige le comité « Littératures et philosophie » de Labyrinthe. Il termine actuellement une thèse portant sur les « lectures de la possession » à l'Université 
Bordeaux-III, où il est enseignant. Au Centre d'études féminines de Paris-VIII, il prépare une étude sur l'amitié et le pathos dans la philosophie. 